\title{
Mini Thoracotomy Approach to Upper Thoracic Spine
}

\author{
Hedaya Hendam1, Hatem El-Samouly', Hamdy M. Behairy², Medhat Noaman³, \\ Gamal Abd Elshafy ${ }^{4}$
}

\author{
${ }^{1}$ Neurosurgery Department, Faculty of Medicine, Al Azhar University, Cairo, Egypt \\ ${ }^{2}$ Department of Neurosurgery, Al-Azhar University, Cairo, Egypt \\ ${ }^{3}$ Anesthesia Department, Faculty of Medicine, Al Azhar University, Cairo, Egypt \\ ${ }^{4}$ Cardiothoracic Department, Faculty of Medicine, Al Azhar University, Cairo, Egypt \\ Email: dr.hamdybehairy@hotmail.com
}

How to cite this paper: Hendam, $H$., El-Samouly, H., Behairy, H.M., Noaman, M. and Elshafy, G.A. (2018) Mini Thoracotomy Approach to Upper Thoracic Spine. Neuroscience \& Medicine, 9, 9-15. https://doi.org/10.4236/nm.2018.91002

Received: December 17, 2017

Accepted: March 3, 2018

Published: March 6, 2018

Copyright (c) 2018 by authors and Scientific Research Publishing Inc. This work is licensed under the Creative Commons Attribution International License (CC BY 4.0).

http://creativecommons.org/licenses/by/4.0/

\section{cc) (i) Open Access}

\begin{abstract}
Upper thoracic spine lesions are characterized by delayed diagnosis due to nonspecific symptoms and its anterior aspect (T1 - T4) is difficult to be approached surgically. This retrospective clinical study was done to assess the efficacy of mini thoracotomy approach in management of these lesions. We studied 14 cases with upper thoracic spine different pathological lesions at levels (T1 - T4). These lesions were indicated for anterior approach surgery. Radiological assessment included plain X-ray, MRI and CT scan thoracic spine. The anaesthesia was specifically selective intubation and unilateral ventilation. All patients were operated upon through mini inter-costal thoracotomy approach (left sided in 13 patients and right sided in 1patient). Out of 14 patients there were 7 males and 7 females. The age range was 20 - 55 years (mean 38.7 years). Preoperative symptoms included vague nonspecific upper thoracic pain in 11 patients, lower limbs weakness in 6 patients, sphincteric disturbance in 5 patients, upper thoracic kyphotic deformity in 4 patients, severe brachialgia in 3 patients and neck pain in 2 cases. The operative time was ranged from 90 - 210 minutes (mean $152 \mathrm{~m}$ ). Blood loss ranged from 250 to $750 \mathrm{cc}$ (mean $464 \mathrm{cc}$ ). Chest tube drainage was inserted in all patients. There were no intra-operative or post-operative surgically related complications and no patients needed ICU admission. The lesions were neoplasms in 8 patients, traumatic fracture dislocation in 3 cases, tuberculous spondylodiscitis in 2 cases and degenerative disc prolapse in 1 case. All patients improved post-operative as regard their pre-operative complaints except one patient. During the follow up period, no mortality was recorded. In conclusion, mini thoracotomy approach seems to be ideal only for patients with mono- or bi-segmental pathology involving the upper thoracic spine.
\end{abstract}




\section{Keywords}

Minithoracotomy, Upper Dorsal Spine

\section{Introduction}

The anterior aspect of the upper thoracic spine (T1 - T4) is a difficult area to be approached surgically. The normal upper thoracic kyphosis produces deep surgical field. The thoracic cage, sternum, clavicle, and the major blood vessels in front of the spine impose limitations for anterior approach. Pathological involvement of the upper thoracic spine usually results in collapse of the vertebral bodies increases the kyphosis and adds further difficulties [1]. Diagnosis is often delayed in patients with upper thoracic spine problems because of the difficulties in obtaining good radiological images. Clinicians may overlook the lesions in absence of cord compression deficits as those patients often presented by nonspecific complaints such as vague thoracic back pain. Selection of surgical approach depends on the extension of the lesion, instrumentation needed and reconstruction of the spine. The most important goal when choosing a surgical approach here is to minimize manipulations on surrounding structures and the already compromised thoracic spinal cord [2].

Approaching upper thoracic spine through right or left mini thoracotomy made at the intercostal space between the $3^{\text {rd }}$ and $4^{\text {th }}$ ribs with sub-periosteal dissection and protection of the inferior neurovascular bundle can expose T1 - T4 levels safely and easily particularly in the presence of kyphosis. This approach is sufficient to deal with different pathologies and recently has gained more popularity as it provides the shortest direct route to upper thoracic spine and leaves the pleura intact [3].

Because the integrity of the pleura is maintained, routine closed chest tube drainage is not necessary [4]. Also retro-pleural approaches do not necessitate deflation of the lung. A smaller incision and less retraction than traditional approaches may reduce post-operative pain and pulmonary related complications [3].

In this study, we tried to evaluate the utility of the less invasive mini thoracotomy approach instead of the often used conventional generous rib removing thoracotomy or posterior risky approaches in management of different pathologies in the so difficult area of the spine "T1 - T4" which always considered as a major challenge for spine surgeons.

\section{Patients and Methods}

This is a retrospective study done at Al-Azhar university hospitals, from October 2011 to October 2015 and involved 14 patients with upper thoracic spine lesions (T1 - T4) whom indicated for antero-lateral surgical approach. Review of the clinical charts of the patients regarding, age, sex, pre-operative clinical presentation, radiological and laboratory investigations, identification of the anatomical 
site of the lesions, surgical procedures, operative complications, post-operative neurological status, histopathological examination, radiotherapy and/or chemotherapy to patients with malignant neoplastic lesions and antibiotics used for infectious lesions. All patients were operated through mini-thoracotomy antero-lateral approach in lateral position. Data related to the surgery as; procedure, duration, blood loss, intra-operative complications, chest tube insertion, intensive care admission, post-operative recovery and delayed complications were collected.

\subsection{Ethical Considerations}

Written informed consents were obtained from all participant patients after full explanation of the surgical technique and that their demographic and clinical data will be included in the study while patient identities will not be included. This study was approved by the ethics committee of our department.

\subsection{Surgical Procedure}

Patients were placed in lateral decubitus position. An axillary roll was placed to minimize the risk of brachial plexus injury. Fluoroscopy was used to confirm and mark the skin incision. The incision was made over the third rib. A sub-periosteal dissection was performed, protecting the inferior neurovascular bundle. The intercostal retractor was placed to open the thorax. Trials of retro-pleural dissection were made. If failed, we went trans-pleural for exploration of the posterior chest wall and vertebral bodies. The lung was retracted with a malleable or egg beater retractor. The spine could be seen through the parietal pleura. The segmental vessels in the planned area of resection were controlled by ligation or coagulation. The proximal 2 to $3 \mathrm{~cm}$ of the head of the rib was sectioned using a high-speed drill or osteotome, and then disarticulated for exposing the intercostal nerve, neural foramen and the pedicle. The discs above and below the pathological vertebrae are incised and removed in an anterior direction away from the cord. A high speed-drill with a cutting burr was used to remove most the ventral aspect of the vertebral body. The anterior cortex and anterior longitudinal ligament are left intact. The posterior cortical margin is thinned down to an egg shell thickness, and fractured by curettes, and then the spinal cord was decompressed. Reconstruction and realignment was done using iliac crest bone graft, bone cement, titanium mesh or expandable intervertebral cage and anterior plate. Closure of the parietal pleura and placement of chest tube, and individual muscles identified and closed in layers.

\section{Results}

This study included 14 patients with upper dorsal spine lesion treated surgically with mini-thoracotomy approach. Seven were males and 7 were females, with the age ranged from 20 years to 55 years, (mean age 38.7 years).

The patients were presented by vague nonspecific upper thoracic spine pain in 
11 patients, lower limbs weakness in 6 patients, sphincter complaints in 5 patients upper thoracic kyphotic deformity in 4 patients, severe brachialgia in 3 patients, neck pain in 2 cases (Table 1 ).

The pre-operative imaging of upper dorsal spine lesions was; plain X-ray "antero-posterior and lateral views" and MRI in all patients. CAT scan was done in 8 patients and isotope scanning in 8 patients. Post-operative imaging was done in the form of plain X-ray in all patients, CT scan in 1 patient and MRI in 1 patient. All patients were operated upon through left antero-lateral intercostal mini-thoracotomy approach in 13 patients and through the right side in one patient. The operative time was ranged from 90 - 210 minutes (mean $152 \mathrm{~m}$ ). The blood loss ranged from 250 - $750 \mathrm{cc}$ (mean $464 \mathrm{cc}$ ). Chest tube drainage was inserted in all patients. After surgery, no patients needed postoperative intensive care unit admission.

Intra-operative complications included lung tears in 2 cases that sutured immediately by the thoracic surgeon, bleeding from subcostal artery in 1 case that was controlled soon by bipolar diathermy.

Post-operative complications included pain in the wound site and radicular pain along upper intercostal nerves that was severe in the start then was ameliorated by administration of pregabaline in addition to non-steroidal anti-inflammatory drugs. Delayed wound healing was noticed in the 2 cases with tuberculous spondylodiscitis. No cases of major vascular or neurological complications. No cases of wound infection.

The pathological diagnosis of the lesions (Table 2) was neoplastic in 8 patients; 3 plasmocytomas, 2 schwannomas, 1 neurofibroma, 1 metastatic adenocarcinoma and 1 osteoblasoma. Other lesions were tuberculous spondylodiscitis in 2 cases, traumatic fracture dislocation in 3 cases and 1 case was degenerative dorsal disc prolapse.

Reconstruction of the upper dorsal spine was done in 10 patients $(71.4 \%)$ through titanium mesh in 4 patients, expandable intervertebral cage in 3 patients, bone graft in 2 patients, and bone cement in 1 patient along with antero-lateral plate fixation.

The patients were improved post-operatively as regard to their pre-operative complaints (pain, motor weakness, sphincter disturbances, and spine deformities), except in 1 patient with degenerative dorsal disc prolapse who

Table 1. Clinical presentation of the studied cases.

\begin{tabular}{ccc}
\hline Clinical presentation & No. & $\%$ \\
\hline Upper thoracic pain & 11 & 78.5 \\
Lower limb weakness & 6 & 42.9 \\
Sphincter disturbances & 5 & 35.7 \\
Kyphotic deformity & 4 & 28.6 \\
Brachialgia & 3 & 21.4 \\
Neck pain & 2 & 14.3
\end{tabular}


Table 2. Pathology of lesions in studied cases.

\begin{tabular}{ccc}
\hline The pathology & Number of cases & Percent \\
\hline Neoplastic & 8 & 57.14 \\
Traumatic Fracture dislocation & 3 & 21.42 \\
Infection Tuberculous spondylodiscitis & 2 & 14.28 \\
Degenerative Dorsal disc prolapse & 1 & 7.14 \\
\hline
\end{tabular}

was the same as preoperative. During the follow up period no mortality was recorded.

\section{Discussion}

Minithoracotomy approach represents a modification of conventional thoracotomy described for the treatment of pathologies of the anterior thoracic spine. As far as our preliminary experience shows, mini-thoracotomy lead to reduction of intra-and post-operative morbidity in the type of diseases treated so far. Trauma to the rib cage and muscles covering the anterolateral part of the thorax is diminished. The post-operative ICU stay is short as is the time in hospital. The same is true for intra-operative blood loss, incision pain, and cosmoses. The approach, however, seems to be ideal only for patients with mono- or bi-segmental pathology. Excision of malignant tumors, decompression of the spinal canal in tumors, spondylitis, and fracture cases can be achieved without major difficulties. The same is true for biopsies or drainage of pre-vertebral abscesses. The most common indication of our approach in this study was neoplastic lesions (8 out of 14), but in Nadir et al. 2008 series [5], 75\% of the patients was trauma fracture. Mean operative time was approximately 2.5 hours ranged from 1.5 to 3.5 hours, the same as in other series, and estimated blood loss was less than 464 $\mathrm{ml}$ ranged from 250 to $750 \mathrm{ml}$. In this study, we don't record post-operative significant complications. Nadir et al. 2008 [5], among his series of 67 patients operated via the anterior approach, he observed four post-operative complications, one empyema, one hemothorax and two wound infection. However, technical skills must be acquired by surgeons not familiar with the use of surgical microscope or endoscope. Exposure is possible but more difficult, in patient in which single-lung ventilation is not possible. Data extrapolated largely from the cardiothoracic surgical literature indicate infection rates, following median sternotomy, ranging from $0.2 \%$ to $10 \%$ with associated morbidity and mortality rates as high as $25 \%$ [6] [7] [8]. We believe that access to the upper thoracic spine using a less invasive approach such as currently described should be strongly considered in patients, especially, to reduce intra-operative complications. Our cases, show that we can comfortably place a 2-level strut graft or intervertebral device, an anterior plate, and screw at $\mathrm{T} 1$ and down to $\mathrm{T} 5$, but other approaches to the levels cannot been able to secure an anterior plate in the patient below T3 as in technical notes of Fred et al. 2011 [9]. Thus, careful pre-operative planning 
needs to be performed in selecting lesions that can be accesses safely using our approach. We prefer left side thoracotomy in our study, as Nadir et al. 2008, [5] Walkins et al. 2006 [10], and Thongtrongam et al. 2006 [11], to face the lesion with the right hand of the surgeon, and the easy mobilization of the descending aorta if required in anterior approach.

In our study, we have 2 cases of tuberculosis presented by spinal cord compression, kyphotic deformity and neurological deficits as in Zhang et al. 2013 series [12]. Both patients treated through mini intercostal thoracotomy approach with successful relieve of spinal cord compression, correction of spinal kyphotic deformity and finally improve the neurological state post-operatively. Although, the ideal surgical procedure for thoracic tuberculosis is still in debate [13]. Anterior debridement with strut graft fusion, combined with anti-tuberculosis chemotherapy is the standard and effective treatment for the thoracic spinal tuberculosis as we do [3] [6] [7] [14].

\section{Limitations}

This study has many limitations. First, the number of patients is small, so, the real outcome of this technique and the possible associating complications needs further studies with greater number of patients and following different techniques in comparison form for generalization of the results. Second, this technique could not be performed in separate spine centers as cardiothoracic intensive care should be available. Third, high expert anesthetist and cardiothoracic surgeon are needed for performing such technique.

\section{Conclusion}

The upper thoracic spine region (T1 - T4) is a difficult and challenging to be approached surgically and the antero-lateral mini thoracotomy approach is considered an ideal surgical approach to this area. This approach had greater advantages as we can through it deal with all indicated pathological lesions with sufficient exposure and reconstruction.

\section{References}

[1] Fung, K., Lam, C. and Law, S. (2001) Anterior Approach to the Upper Thoracic Spine (T2-T4), the Experience of Seven Cases. Hong Kong Journal, 5, 95-102.

[2] Oppenlander, M., Clork, J. and Kalyuos, J. (2011) Surgical Management and Clinical Outcome of Multiple Level Symptomatic Herniated Thoracic Discs. Journal of Neurosurgery: Spine.

[3] Hiroyuki, Y. (2014) Surgical Treatment for Thoracic Disc Herniation. Spine, 39, E406-E412.

[4] Angevn, P. and Mc Cormick, P. (2001) Retrolpeural Thoracotomy. Technical Note. Neurosurgical Focus, 10, ecp 1.

[5] Nadir, A., Sahin, E., Ozum, U., Korado, O., Tezeren, G. and Kaptan, M. (2008) Thoracotomy in Spine Surgery. Journal of Thoracic and Cardiovascular Surgery, 56, 482-484. 
[6] Landes, G., Harris, G., Sampalis, J., Brutus, J., Cordoba, C. and Ciaburro, H. (2007) Outcomes in the Management of Sternal Dehiscence by Plastic Surgery: A Ten Years' Review in One University Center. Annals of Plastic Surgery, 59, 659-666. https://doi.org/10.1097/SAP.0b013e31803b370b

[7] Li, M., Du, J., Meng, H., Wong, Z. and Lou, Z. (2011) One Stage Surgical Management for Thoracic Tuberculosis by Anterior Debridement, Decompression, and Autologous Rib Graft and Instrumentations. The Spine Journal, 11, 726-733.

[8] Mulpuri, K., Le Blance, J., Reilly, C., Poskitt, K., Choit, R. and Sahajpl, V. (2005) Sternal Split Approach to the Cervicothoracic Junction in Children. Spine, 30, E305-E310. https://doi.org/10.1097/01.brs.0000164267.30422.a9

[9] Fred, C., Michael, W. and Groff, A. (2011) An Anterior Approach to Spinal Pathology to the Upper Thoracic Spine through a Partial Manubriotomy. Journal of Neurosurgery: Spine, 15, 467-471. https://doi.org/10.3171/2011.6.SPINE11189

[10] Walkins, R. (2006) Thoracic Spine Anterior. In: Herkowit, H.N., Garfin, S.R., Eismant, F.J., Bell, G.R., Balderston, R.A., Eds., Rothman-Simeone. Spine Surgery, 5th Edition, Philadelphia Sunders Elsevier, 290-307.

[11] Thongtrongam, I., Lept, N., PorK, J. and Kim, D. (2006) Thoracic and Thoracolumbar Fractures. 9th Edition, Sunders Elsevier, Philadelphia, 352-363.

[12] Zhang, H., Sheng, B., Tong, M., Guo, C. and Lius, S. (2013) One Stage Surgical Treatment to Upper Thoracic Spinal Tuberculosis by Internal Fixation, Debridement, and Combined Interbody and Posterior Fusion via Posterior only Approach. European Spine Journal, 22, 616-623. https://doi.org/10.1007/s00586-012-2470-1

[13] Zhang, H., Li, J., Zhaoo, S., Shao, Y. and Lius, H. (2012) Surgocal Management of Thoracic Spine Tuberculosis in Elderly. Archives of Orthopedic and Trauma Surgery, 132, 1717. https://doi.org/10.1007/s00402-012-1618-0

[14] Garg, B., Kandwal, P., Nogoroja. U., Goswomi, A. and Joyaswol, A. (2012) Anterior versus Posterior Procedure for Surgical Treatment of Thoraco-Lumbar Tuberculosis. A Retrospective Analysis. Indian Journal of Orthopaedics, 46, 165. 